\title{
The association of XRCC3 Thr241Met genetic variant with risk of prostate cancer: a meta-analysis
}

\author{
Gao Xuan, Ying Hui ,He Fang
}

College of Life Science, Anhui Normal University, Wuhu 241000, Hubei Province, People’s Republic of China

\begin{abstract}
Background: Previous studies suggest that the X-ray repair cross-complementing group 3 gene (XRCC3) Thr241Met genetic variant could be potentially associated with the risk of prostate cancer. However, results from these published studies were conflicting rather than conclusive.

Objectives:This meta-analysis aimed to conduct a better understanding of the effects of XRCC3 Thr241Met genetic variant on prostate cancer risk.

Methods: We identified three eligible studies, 499 prostate cancer cases and 571 controls.

Results: Overall, significant associations were detected in the heterozygote comparison genetic model (CT versus (vs.) CC: $\mathrm{OR}=0.71,95 \% \mathrm{CI} 0.53-0.94, \mathrm{Z}=2.38, \mathrm{p}=0.017$ ), and the dominant genetic model (TT/CT vs. CC: $\mathrm{OR}=0.74,95 \% \mathrm{CI} 0.57-0.98, \mathrm{Z}=2.11, \mathrm{p}=0.035$ ). In the subgroup analysis by ethnicities, we found that this genetic variant was significantly associated with the decrease risk of prostate cancer in Caucasians for heterozygote comparison genetic model (CT vs. CC: $\mathrm{OR}=0.66,95 \% \mathrm{CI} 0.44-0.98, \mathrm{Z}=2.04, \mathrm{p}=0.042$ ).

No publication bias was found in this study.

Conclusions: Results from this meta-analysis indicate that the XRCC3 Thr241Met genetic variant is associated with prostate cancer risk.
\end{abstract}

Keywords: Prostate cancer; XRCC3 gene; Genetic variant; Meta-analysis

DOI: http://dx.doi.org/10.4314/ahs.v15i1.16

\section{Introduction}

Prostate cancer is the most common malignancy of men in the world, accounting for $10 \%$ of men cancerrelated mortality ${ }^{1,2}$. The etiology of prostate cancer is largely unknown, although genetic and environmental factors might increase risk of prostate can$\operatorname{cer}^{2-6}$. The X-ray repair cross-complementing group 3 (XRCC3) is one of the DNA repair genes, and is an important candidate gene for mediating the genetic influence on prostate cancer $^{7-13}$. The $\mathrm{C} 18067 \mathrm{~T}$ genetic variant in XRCC3 gene at exon 7(C>T, rs861539), one of the most studied functional genetic variants,
Corresponding author:
Gao Xuan,
College of Life Science,
Anhui Normal University, No 1 Beijin
East Road, Wuhu 241000, Hubei Province,
People's Republic of China.
Tel: +86-0553-5910027;
Fax: +86-0553-5910027
E-mail: xuangao_12@sina.com

results from a $\mathrm{C}$ to $\mathrm{T}$ mutation and causes the substitution of Threonine (Thr) to Methionine (Met) at codons 241 (p.Thr241Met), has been potentially associated with the risk of prostate cancer ${ }^{7-10}$. However, results from published studies were conflicting rather than conclusive. Therefore, to clarify the effects of XRCC3 Thr241Met genetic variant on prostate cancer risk, we conducted a meta-analysis of all available published studies to date.

\section{Materials and methods \\ Publication search}

Pubmed, Excerpta Medica Database (EMBASE), and Chinese National Knowledge Infrastructure (CNKI) databases were searched using the search terms: "prostate cancer/neoplasm", "XRCC3",m“Thr241Met", and "rs8761539" (the last search was updated on June 2014). Publication searching was utilized without limitation on language and publication date. Two investigators searched the publication literature and extracted data independently.

\section{Inclusion, exclusion criteria and Data extraction}

For inclusion criteria in the present meta-analysis, the selected eligible articles had to provide informa- 
tion as follows: ${ }^{1}$ using a case-control design; ${ }^{2}$ evaluation heterogeneity was not significant among the studies ${ }^{18}$. of XRCC 3 Thr $241 \mathrm{Met}$ genetic variant with the risk of Otherwise, the random-effects model (the DerS the odds ratios (ORs) and $95 \%$ confidence intervals funnel plot and Egger's linear regression methods were (CIs); ${ }^{4}$ only full-text articles were included. The exclu- $\quad$ used to assess the publication bias ${ }^{20,21}$. All analyses were sion criteria of articles were as followed: ${ }^{1}$ duplication; analyzed by the STATA software (version 11.0; STATA 2 no usable data was provided; ${ }^{3}$ abstract, comment, Corporation, College Station, TX, USA). P-values letters, and review. For each eligible case-control arti- $<0.05$ were defined as statistically significant level. cles, the following information was collected: the first author's name, publishing year, country, ethnicities, numbers of cases and controls, genotyping methods, numbers of allele and genotype.

\section{Statistical analysis}

The strength of the association of XRCC3 Thr241Met genetic variant with the risk of prostate cancer was assessed by the pooled ORs with their $95 \%$ CIs. Subgroup analyses were evaluated by ethnicities.

The significance of pooled ORs was determined by the Z-test. The heterogeneity assumption was evaluated by the chi-square-based Q-test ${ }^{14,15}$ and the I2 index 16. I2 index $<50 \%$ and/or P-value $>0.10$ for Q-test indicated a lack of heterogeneity among the studies ${ }^{17}$ The fixed effect model (the Mantel-Haenszel method) was utilized to calculate the pooled ORs when the

\section{Table 1. The characteristics of eligible studies included in this meta-analysis.}

\begin{tabular}{|c|c|c|c|c|c|c|c|c|c|c|c|}
\hline \multirow[t]{2}{*}{ First author } & \multirow[t]{2}{*}{ Year } & \multirow[t]{2}{*}{ Country } & \multirow[t]{2}{*}{ Ethnicity } & \multirow{2}{*}{$\begin{array}{l}\text { Genotyping } \\
\text { methods }\end{array}$} & \multirow{2}{*}{$\begin{array}{l}\text { No. } \\
\text { (cases/controls) }\end{array}$} & \multicolumn{3}{|c|}{ Case $(\%)$} & \multicolumn{3}{|c|}{ Control(\%) } \\
\hline & & & & & & $\mathrm{CC}$ & CT & TT & $\mathrm{CC}$ & CT & TT \\
\hline Ritchey & 2005 & USA & Caucasians & MALDI-TOF & $159 / 247$ & 139 & 17 & 3 & 214 & 31 & 2 \\
\hline Mandal & 2010 & India & Asians & PCR-RFLP & 224/192 & 137 & 78 & 9 & 103 & 77 & 12 \\
\hline Dhillon & 2011 & Australia & Caucasians & PCR-RFLP & 116/132 & 60 & 44 & 12 & 54 & 72 & 6 \\
\hline
\end{tabular}

MALDI-TOF Matrix-Assisted Laser Desorption/Ionization Time of Flight Mass Spectrometry:PCR-RFIP.Polymerase Chain

Reaction-Restriction Fragment Length Polymorphism

\section{Meta-analysis}

Table 2 summarized the association strength between

XRCC3 Thr241Met genetic variant and the risk of

prostate cancer. In the overall, significant associa- tions were detected in heterozygote comparison renetic model (CT versus (vs.) CC: $\mathrm{OR}=0.71,95 \%$

CI 0.53-0.94, Z =2.38, P = 0.017, Table 2, Figure 1), and dominant genetic model (T'T/CT vs. CC: OR $=$ $0.74,95 \%$ CI $0.57-0.98, \mathrm{Z}=2.11, \mathrm{P}=0.035$, Table 2 .
Table 2. The meta-analysis of $X R C C 3 \mathrm{Thr} 241 \mathrm{Met}$ genetic variant and prostate cancer risk

\begin{tabular}{|c|c|c|c|c|c|c|c|c|c|}
\hline \multirow[t]{2}{*}{ Comparisons } & \multirow[t]{2}{*}{ Population } & \multicolumn{3}{|c|}{ Test of association } & \multirow[b]{2}{*}{ P-value } & \multirow[b]{2}{*}{ Model } & \multicolumn{3}{|c|}{ Test of Heterogeneity } \\
\hline & & $\mathbf{N}$ & OR $(95 \% \mathrm{CI})$ & $\bar{Z}$ & & & $\chi^{2}$ & P-value & $I^{2}(\%)$ \\
\hline \multirow[t]{3}{*}{ TT vs. $\mathrm{CC}$} & Overall & 3 & $1.05(0.56-1.94)$ & 0.14 & 0.887 & $\mathrm{~F}$ & 3.58 & 0.167 & 44.1 \\
\hline & Asians & 1 & $0.56(0.23-1.39)$ & 1.25 & 0.213 & $\mathrm{~F}$ & & & \\
\hline & Caucasians & 2 & $1.91(0.77-4.73)$ & 1.40 & 0.161 & $\mathrm{~F}$ & 0.05 & 0.815 & 0 \\
\hline \multirow[t]{3}{*}{ CT vs. $\mathrm{CC}$} & $\begin{array}{l}\text { Overall } \\
\text { S }\end{array}$ & 3 & $0.71(0.53-0.94)$ & 2.38 & 0.017 & $\mathrm{~F}$ & 1.31 & 0.519 & 0 \\
\hline & & 1 & $0.76(0.51-1.14)$ & 1.32 & 0.188 & $\mathrm{~F}$ & & & - \\
\hline & Caucasians & 2 & $0.66(0.44-0.98)$ & 2.04 & 0.042 & $\mathrm{~F}$ & 1.05 & 0.305 & 4.9 \\
\hline \multirow[t]{3}{*}{ TT/CT vs. CC } & Overall & 3 & $0.74(0.57-0.98)$ & 2.11 & 0.035 & $\mathrm{~F}$ & 0.86 & 0.650 & 0 \\
\hline & Asians & 1 & $0.74(0.50-1.09)$ & 1.54 & 0.122 & $\mathrm{~F}$ & & - & - \\
\hline & Caucasians & 2 & $0.75(0.51-1.11)$ & 1.44 & 0.150 & $\mathrm{~F}$ & 0.85 & 0.355 & 0 \\
\hline \multirow[t]{3}{*}{ TT vs. $\mathrm{CT} / \mathrm{CC}$} & Overall & 3 & $1.37(0.51-3.73)$ & 0.62 & 0.534 & $\mathrm{R}$ & 4.43 & 0.109 & 54.8 \\
\hline & Asians & 1 & $0.63(0.26-1.52)$ & 1.03 & 0.304 & $\mathrm{~F}$ & & & - \\
\hline & Caucasians & 2 & $2.41(1.00-5.82)$ & 1.95 & 0.051 & $\mathrm{~F}$ & 0 & 0.979 & \\
\hline \multirow[t]{3}{*}{ T vs. C } & Overall & 3 & $0.85(0.68-1.06)$ & 1.47 & 0.142 & $\mathrm{~F}$ & 0.91 & 0.634 & 0 \\
\hline & Asians & 1 & $0.76(0.56-1.05)$ & 1.65 & 0.100 & $\mathrm{~F}$ & & & \\
\hline & Caucasians & 2 & $0.93(0.68-1.27)$ & 0.45 & 0.653 & $\mathrm{~F}$ & 0.17 & 0.680 & 0 \\
\hline
\end{tabular}

$\mathrm{N}$, number of comparisons; OR, odds ratio; CI, confidence interval; vs., versus; TT vs. CC: Homozygote comparison

CT vs. CC: Heterozygote comparison; TT/CT vs. CC: Dominant model; TT vs. CT/CC: Recessive model; T vs. C:

Allele comparison; R, random effect model; F, fixed effect model; Random effect model was chosen when P-value $<0.10$

and $/$ or $\mathrm{I}^{2}>50 \%$ for heterogeneity test; otherwise fixed effect model was used.

Figure 1. Forest plots of the association between $X R C C 3$ Thr241Met genetic variant and prostate cancer risk (Heterozygote (CT versus. $\mathrm{CC})$ ).

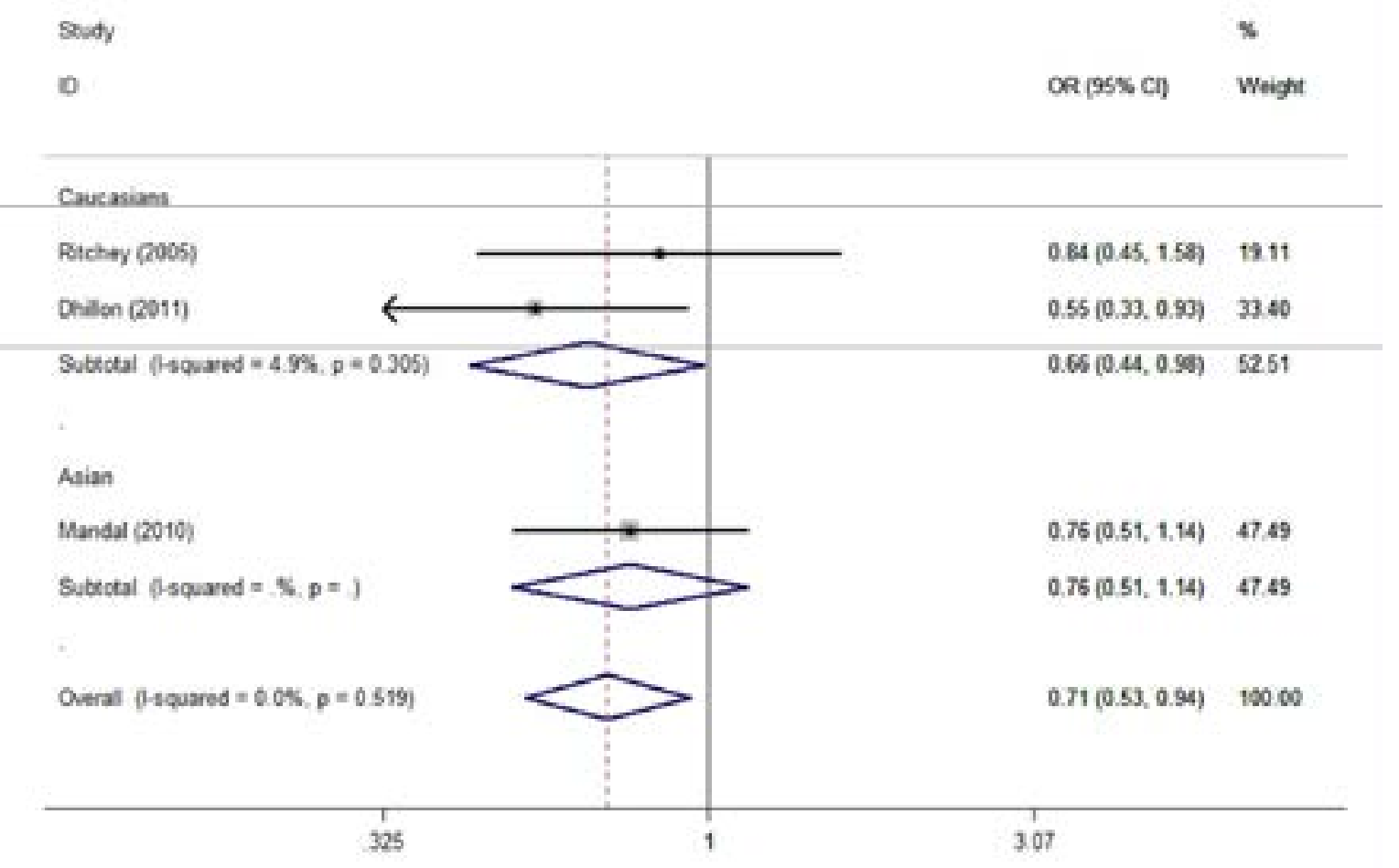


In the subgroup analysis by ethnicities, we found that $=0.042$, Table 2 ). Our data indicated that there were no the XRCC3 Thr241Met genetic variant was significantly significant associations between XRCC3 Thr241Met associated with the decrease risk of prostate cancer in genetic variant and prostate cancer risk in other geCaucasians for heterozygote comparison genetic model netic models (All P-values $>0.05$, Table 2). No evi(CT vs. CC: $\mathrm{OR}=0.66,95 \%$ CI 0.44-0.98, $\mathrm{Z}=2.04, \mathrm{P}$ dence of publication bias was found in all comparison genetic models (All P-values > 0.05)

Figure 2. Begg's funnel plot for publication bias test (Heterozygote comparison (CT versus. CC)).

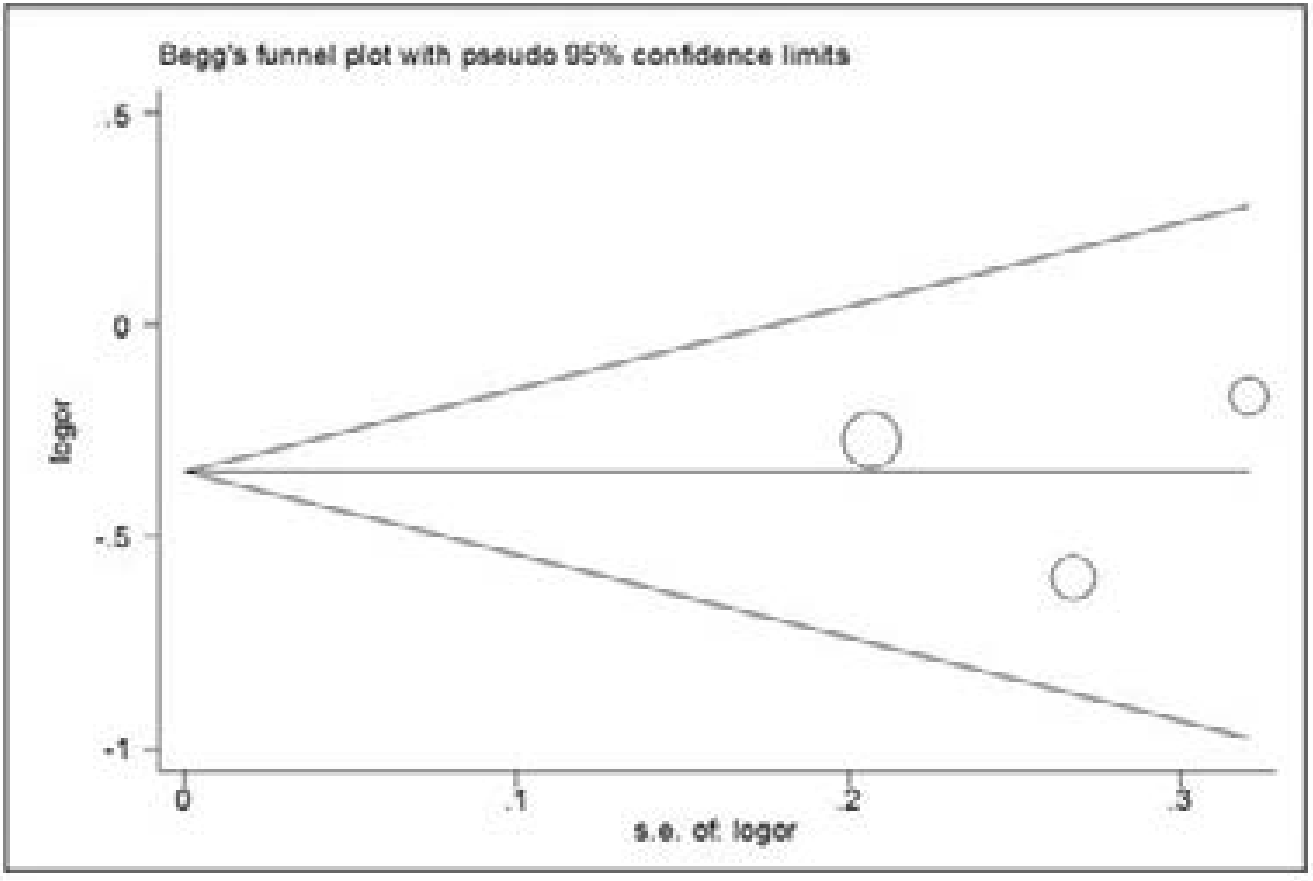

\section{Discussion}

analysis, including 499 prostate cancer cases and 57

Emerging evidence suggest that the XRCC3 is one of the most important candidate genes for influencing the risk of prostate cancer, carried out to investigate the potentinl association XRCC3 Thr241Met genetic variant with the risk of prostate cancer. Ritchey and colleagues reported that XRCC3 Thr241Met genetic variant showed no significant associations with the risk of prostate cancer, while a significant interaction was found for XRCC3 Thr241Met genetic variant and consumption of total preserved foods ${ }^{7}$. Mandal suggested that no significant association of XRCC3 Thr241Met genetic variant genotypes with the risk of prostate cancer was observed ${ }^{8}$. Dhillon demonstrated that there was Some advantages of this meta-analysis should be adno association between the XRCC3 Thr241Met genetic dressed. First, a strict searching strategy to enroll all variant and prostate cancer risk ${ }^{9}$. The present meta- the possible eligible articles as much as possible was conducted. Second, all included articles had acceptable quality. Third, the whole pooled findings are unbiased. However, some limitations of this meta-analysis should be addressed. Firstly, only three eligible articles were eventually enrolled in this meta-analysis. Secondly, the enrolled articles only concerned about Asian and Caucasians, not mentioned about other ethnicities. Thirdly, only published articles were enrolled, unpublished articles were not enrolled in this study.

\section{Conclusion}

This meta-analysis provided evidence of the association of XRCC3 Thr241Met genetic variant with risk of prostate cancer. More well-designed studies in large populations should be carried out to confirm these findings.

\section{Acknowledgements}

This work was supported by National Natural Science Foundation of China (31101148).

\section{Conflict of Interest}

None.

\section{References}

1. Jemal A, Siegel R, Ward E, Hao Y, Xu J, Murray T, Thun MJ. Cancer statistics, 2008. CA CancerJ Clin. 2008; 58: 71-96.

2. Geng J, Zhang Q, Zhu C, Wang J, Chen L. XRCC1 genetic polymorphism Arg399Gln and prostate cancer risk: a meta-analysis. Urology. 2009; 74: 648-653.

3. Pienta KJ, Esper PS. Risk factors for prostate cancer. Ann Intern Med. 1993; 118: 793-803.

. Lichtenstein P, Holm NV, Verkasalo PK, Iliadou A Kaprio J, Koskenvuo M, Pukkala E, Skytthe A, Hemminki K. Environmental and heritable factors in the causation of cancer--analyses of cohorts of twin from Sweden, Denmark, and Finland. N Engl J Med. 2000; 343: 78-85.

5. Schaid DJ. The complex genetic epidemiology of prostate cancer. Hum Mol Genet. 2004; 13 Spec No 1: R103-121.

6. Wei B, Zhou Y, Xu Z, Ruan J, Zhu M, Jin K, Zhou

D, Hu Q, Wang Q, Wang Z, Yan Z. XRCC1 Arg399Gln and Arg194Trp polymorphisms in prostate cance risk: a meta-analysis. Prostate Cancer Prostatic Dis. 2011; 14: 225-231 $820-826$ 
18. Mantel N, Haenszel W. Statistical aspects of the analysis of data from retrospective studies of disease. J Natl Cancer Inst. 1959; 22: 719-748.

19. DerSimonian R, Laird N. Meta-analysis in clinical trials. Control Clin Trials. 1986; 7: 177-188.
20. Egger M, Davey Smith G, Schneider M, Minder C. Bias in meta-analysis detected by a simple, graphical test. BMJ. 1997; 315: 629-634.

21. Begg CB, Mazumdar M. Operating characteristics of a rank correlation test for publication bias. Biometrics. 1994; 50: 1088-1101. 